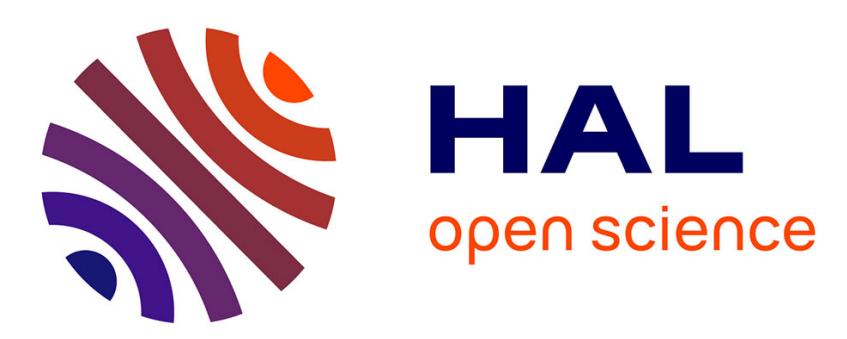

\title{
Measurement of the working parameters of an air-post vertical-cavity surface-emitting laser
}

Stephane Barland, Piernicola Spinicelli, Giovanni Giacomelli, Francesco Marin

\section{To cite this version:}

Stephane Barland, Piernicola Spinicelli, Giovanni Giacomelli, Francesco Marin. Measurement of the working parameters of an air-post vertical-cavity surface-emitting laser. IEEE Journal of Quantum Electronics, 2005, 41 (10), pp.1235 - 1243. 10.1109/JQE.2005.854317 . hal-00263779

\section{HAL Id: hal-00263779 \\ https://hal.science/hal-00263779}

Submitted on 13 Mar 2008

HAL is a multi-disciplinary open access archive for the deposit and dissemination of scientific research documents, whether they are published or not. The documents may come from teaching and research institutions in France or abroad, or from public or private research centers.
L'archive ouverte pluridisciplinaire $\mathbf{H A L}$, est destinée au dépôt et à la diffusion de documents scientifiques de niveau recherche, publiés ou non, émanant des établissements d'enseignement et de recherche français ou étrangers, des laboratoires publics ou privés. 


\title{
Measurement of the Working Parameters of an Air-Post Vertical-Cavity Surface-Emitting Laser
}

\author{
Stephane Barland, Piernicola Spinicelli, Giovanni Giacomelli, and Francesco Marin
}

\begin{abstract}
We present a complete experimental evaluation of the effective parameters necessary to describe the dynamical behavior of an air-post vertical-cavity surface-emitting lasers, on the basis of theoretical equations which are also derived in this paper. The experimental investigation is composed of several steps, including power versus current measurement, noise spectrum analysis, linewidth evaluation. The complete set of parameters derived, in particular the linewidth-enhancement factor and the spontaneous emission factor, is particularly important for accurate comparisons of theoretical models on the laser dynamics with experiments.
\end{abstract}

Index Terms-Amplitude noise spectrum, lineshape, linewidth enhancement factor, relative intensity noise (RIN), semiconductor lasers, spontaneous emission, vertical-cavity surface-emitting lasers (VCSELs).

\section{INTRODUCTION}

$\mathbf{S}$ EMICONDUCTOR lasers are extremely important both for application and basic research on laser physics, quantum optics and nonlinear physics. With respect to other laser sources, their main working parameters are more difficult to be specified, depending on a large variety of structures that are exploited. Direct and complete measurements of such parameters are not frequent and theoretical modeling often uses general, reasonable values even when direct comparison between experiments and theoretical findings are performed and presented. Moreover, some parameters are very critical in determining the laser behavior, such as the amount of spontaneous emission noise and the linewidth enhancement factor $\alpha$ [1]. Concerning the former, the assumed values are usually derived from theoretical calculations, or from assumed average laser characteristics, that can, however, vary over a couple of orders of magnitude. The latter is particularly important both for applications (being at the origin of sensitivity to back-scattered light, linewidth broadening and

Manuscript received March 8, 2005; revised May 24, 2005. This work was supported in part by the European Commission through the RTN network VISTA under Contract HPRN-CT-2000-00034.

S. Barland was with the Istituto Nazionale per la Fisica della Materia (INFM), University of Florence, 50019 Florence, Italy. He is now with Institut Non-Linéaire de Nice, UMR 6618 CNRS, 06560 Valbonne, France (e-mail: Stephane.Barland@inln.cnrs.fr).

P. Spinicelli is with the Physics Department, University of Florence, 50019 Florence, Italy (e-mail: spinicelli@inwind.it).

G. Giacomelli is with the Istituto Sistemi Complessi-CNR, 50125 Florence, Italy, and also with the Istituto Nazionale per la Fisica della Materia (INFM), University of Florence, 50019 Florence, Italy (e-mail: gianni@inoa.it).

F. Marin is with the Physics Department and Istituto Nazionale per la Fisica della Materia (INFM), University of Florence, 50019 Florence, Italy, and also with European Laboratory for Non-Linear Spectroscopy, 50019 Florence, Italy. (e-mail: marin@fi.infn.it).

Digital Object Identifier 10.1109/JQE.2005.854317 frequency noise enhancement, chirp) and for investigations of nonlinear dynamical phenomena. Indeed, $\alpha$ strongly affects the critical sensitivity of semiconductor laser to optical feedback, which is the basic ingredient of several experiments investigating peculiar dynamic behaviors.

Measurements of $\alpha$ are performed in several ways, none of which is quite simple and whose accuracy is often difficult to evaluate. An overview of the earlier works was published in [2]. Among the measurement methods of the "device $\alpha$ " (different from the "material $\alpha$ "), we mention the FM/AM modulation, the measurement of the linewidth, and the recently improved passive optical feedback method [3]. Modulation techniques require high speed current driving and detectors, and it can depend in a non trivial way on the device electronic response. In our case, we obtained unclear results due to the low frequency cutoff of the laser case. The derivation of $\alpha$ from the linewidth is a very exploited method. If we just consider the rather recent vertical-cavity surface-emitting lasers (VCSELs), such measurements are described, e.g., in [4]-[8]. In all these reports (except [7]), however, some of the parameters included in the modified Schawlow-Townes expression of the linewidth [1], [9] are calculated from semiconductor laser theory assuming data about the laser structure.

In the present work, we will instead describe a complete experimental evaluation of all the functional parameters necessary for a description of the behavior of a VCSEL. In particular, both $\alpha$ and the spontaneous emission are measured without the help of additional constants, neither theoretically calculated nor assumed.

Our main motivation originates from a study of a peculiar dynamics of semiconductor lasers with moderate optical feedback, operated near threshold: the so-called low-frequency fluctuations (LFF) [10]. Such behavior consists of fast drops of the laser power, followed by slow recovery. This phenomenon is the subject of several theoretical (including numerical simulations) and experimental works, but it is not yet fully understood. For such a study, VCSELs are particularly important, since they show single-mode LFF [11], without the additional complication of multi-mode dynamics [12]. Numerical simulations that we have performed using typical VCSEL parameters have shown that the experimental signal is well reproduced only for unusually high values of $\alpha$ [13]. The same observation is reported by other authors [14]. Moreover, the role of spontaneous emission noise in the dynamics is not clear. Therefore, we have decided to define a set of effective parameters (not necessarily coincident with microscopic quantities) that determine the laser behavior and that can be completely measured by a well defined series of experiments. In this way it will be possible to directly compare the 
observed phenomena with numerical simulations using an accurate choice of the critical parameters, very close to the real system and, therefore, evaluate more reliably whether the observed phenomena are well described.

Our purpose with this work is providing both a complete measurement procedure, and a set of values that will increase the data base of parameters exploitable by theoretical works, which are often limited to general guesses due to the poor experimental data.

This paper is structured as follows. In Section II, we present our theoretical analysis. We start from general equations and, with some approximations, we obtain standard dynamic equations containing few effective parameters. This part is not really original, but it is necessary to set the notation and clarify the meaning and the limits of the following experimental procedure. In the same Section II-B we derive the observable quantities that allow to infer the parameter values. Our experimental investigation is divided into three different stages: the determination of the power versus injection current $(L-I)$ curve, below and above threshold; the analysis of the intensity noise spectra for different values of the current; the measurement of the spectral linewidth as a function of the pump parameter. The experiment is described in Section III, and in Section IV we give and discuss the results, including some additional analysis on the observed variables and data analysis that is suggested by the experimental findings.

\section{THEORETICAL ANALYSIS}

\section{A. General Background}

Our starting-level equations are derived from [15] and are limited to a single, linearly polarized mode with electric field expressed as

$$
\mathrm{E}(\mathbf{r}, t)=\frac{1}{2} \psi(\mathbf{r}) E(t) e^{j 2 \pi \nu t}+\mathrm{c.c}
$$

where $E(t)$ is the slowly varying complex field amplitude, $\nu$ its optical frequency and $\psi(r)$ its transverse profile that we consider normalized so that $\psi^{2}$ has unitary area. ${ }^{1}$ The rate equation for $E(t)$ is

$$
\frac{d E(t)}{d t}=\frac{1+j \alpha}{2}\left[v_{G} \Gamma_{l g} g(t)-\frac{1}{\tau_{p}}\right] E(t)+\sqrt{\frac{R_{\mathrm{sp}}}{2}} \xi(t)
$$

where $g(t)$ is the modal gain, $\alpha$ is the linewidth enhancement factor, $\tau_{p}$ is the photon lifetime, $v_{G}$ is the group velocity, $\Gamma_{l g}$ is the longitudinal confinement factor, $R_{\mathrm{sp}}$ is the spontaneous emission coefficient, and $\xi(t)$ is a complex Gaussian noise term with zero mean and correlation given by $\left\langle\xi(t) \xi^{*}\left(t^{\prime}\right)\right\rangle=$ $2 \delta\left(t-t^{\prime}\right)$.

The modal gain is given by

$$
g(t)=\int|\psi|^{2}(\mathbf{r}) a\left(N(\mathbf{r}, t)-N_{0}\right) d \mathbf{s}
$$

where $a$ is the gain coefficient, $N(\mathbf{r}, t)$ is the carrier density, $N_{0}$ is the carrier density at transparency, and the integration is

\footnotetext{
${ }^{1}$ This normalization is different from the one chosen in [15].
}

over the transverse coordinates. We remark that we are not considering gain saturation, and we are using a linear expansion of the gain versus carrier density around transparency. As we will see, the latter is a good approximation in our operating region. We are also considering an index-guided device, therefore, nonorthogonal modes effects (see, e.g., [16]) can be neglected.

The carrier density variable in the laser active region, assuming axial symmetry, is ruled by the equation

$$
\begin{aligned}
\frac{\partial N(\mathbf{r}, t)}{\partial t}=\frac{D}{r} \frac{\partial}{\partial r}\left(r \frac{\partial N(\mathbf{r}, t)}{\partial r}\right) & -R_{N} \\
& +\frac{J(\mathbf{r})}{e L_{a}}-v_{G} \Gamma_{l g} \frac{\psi^{2}(\mathbf{r})}{L_{a}} g(t) P(t)
\end{aligned}
$$

where $D$ is the carrier diffusion coefficient, $R_{N}$ is the carrier decay rate, $J(\mathbf{r})$ is the injection current density, $e$ is the electron charge, $L_{a}$ is the active region thickness, $P(t)=|E(t)|^{2}$ is the intracavity power in the lasing mode, and we are neglecting the carrier noise.

In an undoped active medium the carrier decay rate can be expressed as a power series of $N$ [17]

$$
R_{N}=A N+B N^{2}+C N^{3} \text {. }
$$

The term proportional to $B$ on the right-hand side of (5) is attributed to radiative spontaneous recombination, while the other two terms are phenomenological contributions that can be associated typically to carrier trapping (linear term) and Auger effect (cubic term). The spontaneous emission rate in (2) is given by

$$
R_{\mathrm{sp}}=\beta L_{a} B \int N^{2}(\mathbf{r}, t) d \mathbf{s}
$$

where $\beta$ is the spontaneous emission factor.

In [15], the authors develop complete analytical and numerical calculations for the steady state of the laser above threshold, even in the presence of several transverse modes, finding the correct spatial distribution of carrier density and electric field. In this work our aim is finding simplified, yet reasonably accurate expressions that can be directly compared with the experiment. At this purpose we neglect the diffusion term in (4) and we consider homogeneous current density and carrier density within the active region. Such assumption is quite reasonable for our laser structure.

We define a dimensionless spatially weighted carrier density, normalized to transparency, as

$$
n(t)=\frac{1}{N_{0}} \int N(\mathbf{r}, t)|\psi|^{2}(\mathbf{r}) d \mathbf{s}
$$

The threshold carrier density is defined as

$$
n_{\mathrm{th}}=1+\frac{1}{v_{G} \Gamma_{l g} a N_{0} \tau_{p}} .
$$

This expression is derived from the steady-state solution of the equation for the electric field amplitude in the absence of spontaneous emission. Multiplying (4) by $\psi^{2}$ and integrating over the transverse coordinates, we obtain

$$
\frac{d n}{d t}=-R_{n}(n)+\frac{J}{e L_{a} N_{0}}-\frac{v_{G} \Gamma_{l g} a}{V}(n-1) P
$$

where

$$
V=\frac{L_{a}}{\int|\psi|^{4} d \mathbf{s}}
$$


represents the effective mode volume, and we write the carrier decay rate as

$$
R_{n}(n)=\widetilde{B}\left[\left(\frac{n}{n_{\mathrm{th}}}\right)^{2}+\widetilde{A}\left(\frac{n}{n_{\mathrm{th}}}\right)+\widetilde{C}\left(\frac{n}{n_{\mathrm{th}}}\right)^{3}\right]
$$

with $\tilde{A}=(A / B)\left(\Gamma_{t r} / N_{0} n_{\text {th }}\right), \widetilde{B}=\left(N_{0} n_{\text {th }}^{2} / \Gamma_{t r}\right) B$, $\widetilde{C}=(C / B)\left(N_{0} n_{\mathrm{th}} / \Gamma_{t r}\right)$ and $\Gamma_{t r}=\int_{\text {Active region }}|\psi|^{2} d \mathbf{s}$ is the transverse confinement factor. $\widetilde{A}$ and $\widetilde{C}$ represent, respectively, the importance of the decay rates due to carrier trapping and to the Auger effect with respect to spontaneous emission, evaluated at threshold.

Near and above threshold the carrier density is not far from $n_{\text {th }}$ and we can replace $R_{n}(n)$ by its first-order expansion. It is useful to define a small-signal carrier lifetime $\tau_{n}$ as [17]

$$
\frac{1}{\tau_{n}}=\left.\frac{d R_{n}}{d n}\right|_{n=n_{\mathrm{th}}}=\frac{\widetilde{B}}{n_{\mathrm{th}}}(2+\widetilde{A}+3 \widetilde{C})
$$

and a modified gain $\eta$ as

$$
\eta=\frac{n_{\mathrm{th}}}{n_{\mathrm{th}}-1} \frac{R_{n}\left(n_{\mathrm{th}}\right)}{\frac{n_{\mathrm{th}}}{\tau_{n}}}=\frac{n_{\mathrm{th}}}{n_{\mathrm{th}}-1} \frac{1+\widetilde{A}+\widetilde{C}}{2+\widetilde{A}+3 \widetilde{C}} .
$$

The steady-state solution of (9) for $n=n_{\mathrm{th}}$ and $P=0$ gives a threshold current density defined as

$$
J_{\mathrm{th}}=R_{n}\left(n_{\mathrm{th}}\right) e L_{a} N_{0}
$$

from which we can define a pump parameter $\mu=J / J_{\mathrm{th}}$. We also normalize the electric field amplitude as

$$
\frac{E(t)}{\sqrt{P_{0}}}=\widetilde{E}=\sqrt{\widetilde{P}} e^{j \phi}
$$

with

$$
P_{0}=\frac{V}{v_{G} \Gamma_{l g} a \tau_{n}}
$$

and where $\phi$ is the electric field phase.

Equation (9) for the carrier density becomes

$$
\frac{d n}{d t}=-R_{n}(n)+\mu R_{n}\left(n_{\mathrm{th}}\right)-(n-1) \frac{\widetilde{P}}{\tau_{n}} .
$$

Well below threshold we can neglect in (17) the stimulated emission, and using (11) we obtain the steady-state equation for stationary value $\bar{n}$ of $n$

$$
\left(\frac{\bar{n}}{n_{\mathrm{th}}}\right)^{2}+\widetilde{A}\left(\frac{\bar{n}}{n_{\mathrm{th}}}\right)+\widetilde{C}\left(\frac{\bar{n}}{n_{\mathrm{th}}}\right)^{3}=(1+\widetilde{A}+\widetilde{C}) \mu .
$$

Near and above threshold, where the first-order expansion of the carrier decay rate around its threshold value can be used, the equations are formally simplified by using the deviation $\tilde{n}$ of the carrier density from transparency, normalized to have unitary value at threshold, i.e.,

$$
\tilde{n}=\frac{n-1}{n_{\text {th }}-1} .
$$

The approximated equation for the new carrier density is written as

$$
\tau_{n} \frac{d \tilde{n}}{d t}=-\tilde{n}+1+\eta(\mu-1)-\tilde{n} \widetilde{P} .
$$

The equation for the normalized electric field amplitude is

$$
\frac{d \widetilde{E}}{d t}=\frac{1+j \alpha}{2} \frac{1}{\tau_{p}}(\tilde{n}-1) \widetilde{E}+\sqrt{\frac{\widetilde{R}_{\mathrm{sp}}}{2}} \xi(t) .
$$

It can be split into two real equations for the normalized power $\widetilde{P}$ and for the phase

$$
\begin{aligned}
& \frac{d \widetilde{P}}{d t}=\frac{1}{\tau_{p}}(\tilde{n}-1) \widetilde{P}+\widetilde{R}_{\mathrm{sp}}+\sqrt{\widetilde{P} \widetilde{R}_{\mathrm{sp}}} \xi_{1}(t) \\
& \frac{d \phi}{d t}=\frac{\alpha}{2} \frac{1}{\tau_{p}}(\tilde{n}-1)+\frac{1}{2} \sqrt{\frac{\widetilde{R}_{\mathrm{sp}}}{\widetilde{P}}} \xi_{2}(t)
\end{aligned}
$$

where $\xi_{1,2}(t)$ are real Gaussian noise terms of zero mean and correlation given by $\left\langle\xi_{i}(t), \xi_{j}\left(t^{\prime}\right)\right\rangle=2 \delta_{i, j} \delta\left(t-t^{\prime}\right)$. The modal spontaneous emission rate used in (21)-(23) is expressed as

$$
\widetilde{R}_{\mathrm{sp}}=\left(\frac{n}{n_{\mathrm{th}}}\right)^{2} \widetilde{R}_{\mathrm{sp}}^{\mathrm{th}} \simeq\left[1+2 \frac{n_{\mathrm{th}}-1}{n_{\mathrm{th}}}(\tilde{n}-1)\right] \widetilde{R}_{\mathrm{sp}}^{\mathrm{th}}
$$

with

$$
\widetilde{R}_{\mathrm{sp}}^{\mathrm{th}}=\frac{\beta \pi r_{0}^{2} L_{a} n_{\mathrm{th}}}{(2+\widetilde{A}+3 \widetilde{C}) \Gamma_{t r} V \tau_{p}\left(n_{\mathrm{th}}-1\right)}
$$

where $r_{0}$ is the mesa radius and the first-order expansion in (24) can be used near and above threshold.

The deterministic steady state of the system is obtained by zeroing the time derivatives in (17) or (20) and (22), and neglecting the noise term in (22).

Near and above threshold, the stationary value $\bar{P}$ of $\widetilde{P}$ is given by (26), shown at the bottom of the page. The asymptotic value of $\bar{P}$ is

$$
\bar{P} \rightarrow \eta(\mu-1)+\tau_{p} \widetilde{R}_{\mathrm{sp}}^{\mathrm{th}} .
$$

The stationary value $\bar{n}$ of $n$ is

$$
\bar{n}=1+\left(n_{\mathrm{th}}-1\right) \frac{1+\eta(\mu-1)}{1+\bar{P}} .
$$

\section{B. Definition of the Observable Quantities}

Equations (17)-(23) contain six functional parameters, namely, $\tau_{p}, \tau_{n}, \alpha, \eta, \widetilde{R}_{\mathrm{sp}}^{\mathrm{th}}$, and $n_{\mathrm{th}}$. These parameters completely determine the laser behavior: the first four are sufficient

$$
\bar{P}=\frac{1}{2}\left[\eta(\mu-1)+\tau_{p} \widetilde{R}_{\mathrm{sp}}^{\mathrm{th}} \frac{2-n_{\mathrm{th}}}{n_{\mathrm{th}}}+\sqrt{\left[\eta(\mu-1)+\tau_{p} \widetilde{R}_{\mathrm{sp}}^{\mathrm{th}} \frac{2-n_{\mathrm{th}}}{n_{\mathrm{th}}}\right]^{2}+4 \tau_{p} \widetilde{R}_{\mathrm{sp}}^{\mathrm{th}}\left[1+2 \eta \frac{n_{\mathrm{th}}-1}{n_{\mathrm{th}}}(\mu-1)\right]} .\right.
$$


for an analysis neglecting the spontaneous emission, which is included with $\widetilde{R}_{\mathrm{sp}}^{\mathrm{th}}$, and in addition $n_{\mathrm{th}}$ is necessary for a faithful description of the region around and below threshold. Our purpose is defining a set of experimental measurements allowing to obtain all there values.

A first useful measurable quantity is the relative intensity noise (RIN) spectrum. Its expression can be found by a standard linearization around the steady state of (20) and (22). The result is [18]

$$
\operatorname{RIN}(\omega)=\frac{4 \widetilde{R}_{\mathrm{sp}}^{\mathrm{th}}}{\bar{P}} \frac{\omega^{2}+\Gamma_{\mathrm{RO}}^{\prime 2}}{\left(\Omega_{\mathrm{RO}}^{2}-\omega^{2}\right)^{2}+\Gamma_{\mathrm{RO}}^{2} \omega^{2}}
$$

where the relaxation oscillation angular frequency $\Omega_{\mathrm{RO}}$, the damping factor $\Gamma_{\mathrm{RO}}$ and $\Gamma_{\mathrm{RO}}^{\prime}$ are given by

$$
\begin{aligned}
& \Omega_{\mathrm{RO}}^{2}=\frac{\bar{P}}{\tau_{n} \tau_{p}}+\frac{\widetilde{R}_{\mathrm{sp}}^{\mathrm{th}}}{\tau_{n} \bar{P}} \\
& \Gamma_{\mathrm{RO}}^{\prime}=\frac{\bar{P}}{\tau_{n}}+\frac{1}{\tau_{n}} \\
& \Gamma_{\mathrm{RO}}=\Gamma_{\mathrm{RO}}^{\prime}+\frac{\widetilde{R}_{\mathrm{sp}}^{\mathrm{th}}}{\bar{P}} .
\end{aligned}
$$

Just above threshold, i.e., for $\eta(\mu-1) \gg \tau_{p} \widetilde{R}_{\mathrm{sp}}^{\mathrm{th}}$, we can replace in the above expressions $\bar{P} \rightarrow \eta(\mu-1)$ and neglect the second term in the expression of $\Omega_{\mathrm{RO}}^{2}$.

A study of $\Omega_{\mathrm{RO}}^{2}$ and $\Gamma_{\mathrm{RO}}^{\prime}$ as a function of the pump parameter will give $\tau_{n}, \tau_{p}$ and $\eta$. In principle, $\Gamma_{\mathrm{RO}}$ should give also $\widetilde{R}_{\mathrm{sp}}^{\mathrm{th}} / \eta$, since the second term on the right-hand side of (32) is still important in the range $\tau_{p} \widetilde{R}_{\mathrm{sp}}^{\mathrm{th}} \ll \eta(\mu-1) \lesssim \tau_{n} \widetilde{R}_{\mathrm{sp}}^{\mathrm{th}}$. However, as we will see, this parameter is hard to obtain from the experimental RIN spectra. Also $\widetilde{R}_{\mathrm{sp}}^{\mathrm{th}}$ could be inferred from the scaling factor of the RIN, but such procedure requires the absolute calibration of the dc and rf response of the fast detector with an accuracy that is difficult to reach.

A further measurable quantity is the output power versus current ( $L-I$ curve). Equation (26) can be written as (33), shown at the bottom of the page, where $\widetilde{I}_{\text {th }}=$ $I_{\mathrm{th}}\left(1-\left(\tau_{p} \widetilde{R}_{\mathrm{sp}}^{\mathrm{th}} / \eta\right)\left(2-n_{\mathrm{th}} / n_{\mathrm{th}}\right)\right) \simeq I_{\mathrm{th}}$ and $I_{\mathrm{th}}$ is the threshold current.

Besides a scaling factor and $\widetilde{I}_{\text {th }}$, the measurements can give factorized parameter $\left(\tau_{p} \widetilde{R}_{\mathrm{sp}}^{\mathrm{th}} / \eta^{2}\right)$. As we have seen, $\eta$ and $\tau_{p}$ are given by the RIN measurements, therefore, the value of $\widetilde{R}_{\mathrm{sp}}^{\mathrm{th}}$ can actually be derived.

The last measurable quantity that we have used to infer the laser functional parameters is the Lorentzian linewidth. From the linearization of (20), (22) and (23) we obtain the single-side phase noise spectral density at low frequencies

$$
S_{\phi \phi}(\omega) \simeq \frac{1}{\omega^{2}} \frac{\widetilde{R}_{\mathrm{sp}}^{\mathrm{th}}}{\bar{P}}\left(1+\alpha^{2}\right)
$$

from which we deduce a Lorentzian lineshape with modified Shawlow-Townes linewidth (FWHM)

$$
\Delta \nu_{S T}=\frac{1}{4 \pi} \frac{\widetilde{R}_{\mathrm{sp}}^{\mathrm{th}}}{\bar{P}}\left(1+\alpha^{2}\right) .
$$

Well above threshold we can use the asymptotic expression for $\bar{P}$, obtaining

$$
\frac{1}{\Delta \nu_{S T}} \rightarrow 4 \pi \frac{\eta(\mu-1)}{\widetilde{R}_{\mathrm{sp}}^{\mathrm{th}}\left(1+\alpha^{2}\right)} .
$$

The slope of $\left(1 / \Delta \nu_{S T}\right)$ versus pump parameter, together with the previously measured $\widetilde{R}_{\mathrm{sp}}^{\mathrm{th}}$ and $\eta$, allows to infer the linewidth enhancement factor $\alpha$.

\section{EXPERIMENTAL SETUP AND MEASUREMENTS}

The laser is an air-post VCSEL made by CSEM [19], [20], operating around $770 \mathrm{~nm}$. The mesa diameter is $9.4 \mu \mathrm{m}$, a ring contact defines the output window with a diameter of $5 \mu \mathrm{m}$, and the active medium is composed of three 8-nm quantum wells [21]. The threshold current is around $2.7 \mathrm{~mA}$ and the laser emission is on a single linearly polarized transverse mode up to at least $6.5 \mathrm{~mA}$ (all the measurements discussed in this paper are performed below this limit).

The laser case temperature is actively stabilized within about $1 \mathrm{mK}$. The pump current is carefully controlled by a home-made battery operated power supply whose current noise, measured on a load resistor, is below $40 \mathrm{pA} \cdot \mathrm{Hz}^{-1 / 2}$ in the range from $1 \mathrm{kHz}$ to $3 \mathrm{MHz}$. We have performed some test measurements by directly biasing the laser with batteries through a few kilo-ohms resistor, to reject the possibility of residual electronic noise effects on the laser linewidth.

The laser emission is collected by slightly tilted lenses, to avoid optical feedback, and sent through an optical isolator. A second isolator is placed before the Fabry-Pérot cavity. We have checked that small movements of the optics do not give any effect in the noise spectrum, nor in the lineshape, which is according to our experience one of the most feedback-sensitive observable features.

The power meter used for the $L-I$ curves, by Newport, uses a wide area silicon head with a nominal accuracy of $2 \%$. The light is coupled to the detector with large numerical aperture optics. For such measurements we are not really interested in the absolute power, but on the accurate shape of the $L-I$ curve. We have thus filtered the laser light in order to operate on a single measurement range of the power meter. The results are reported in Fig. 1.

The intensity noise spectra are recorded by means of two different detectors: an avalanche photodiode (APD) with a bandwidth of $2 \mathrm{GHz}$ and a fiber-coupled p-i-n photodiode (ElectroOptics Technology ET-4000F) with a nominal bandwidth of $10 \mathrm{GHz}$, followed by $32-\mathrm{dB}, 5-\mathrm{GHz}$ amplifiers. An example of

$$
\bar{P}=\frac{\eta}{2 I_{\mathrm{th}}}\left[I-\widetilde{I}_{\mathrm{th}}+\sqrt{\left(I-\widetilde{I}_{\mathrm{th}}\right)^{2}+I_{\mathrm{th}}^{2} \frac{4 \tau_{p} \widetilde{R}_{\mathrm{sp}}^{\mathrm{th}}}{\eta^{2}}\left[1+2 \eta \frac{n_{\mathrm{th}}-1}{n_{\mathrm{th}}}\left(\frac{I}{I_{\mathrm{th}}}-1\right)\right]}\right]
$$



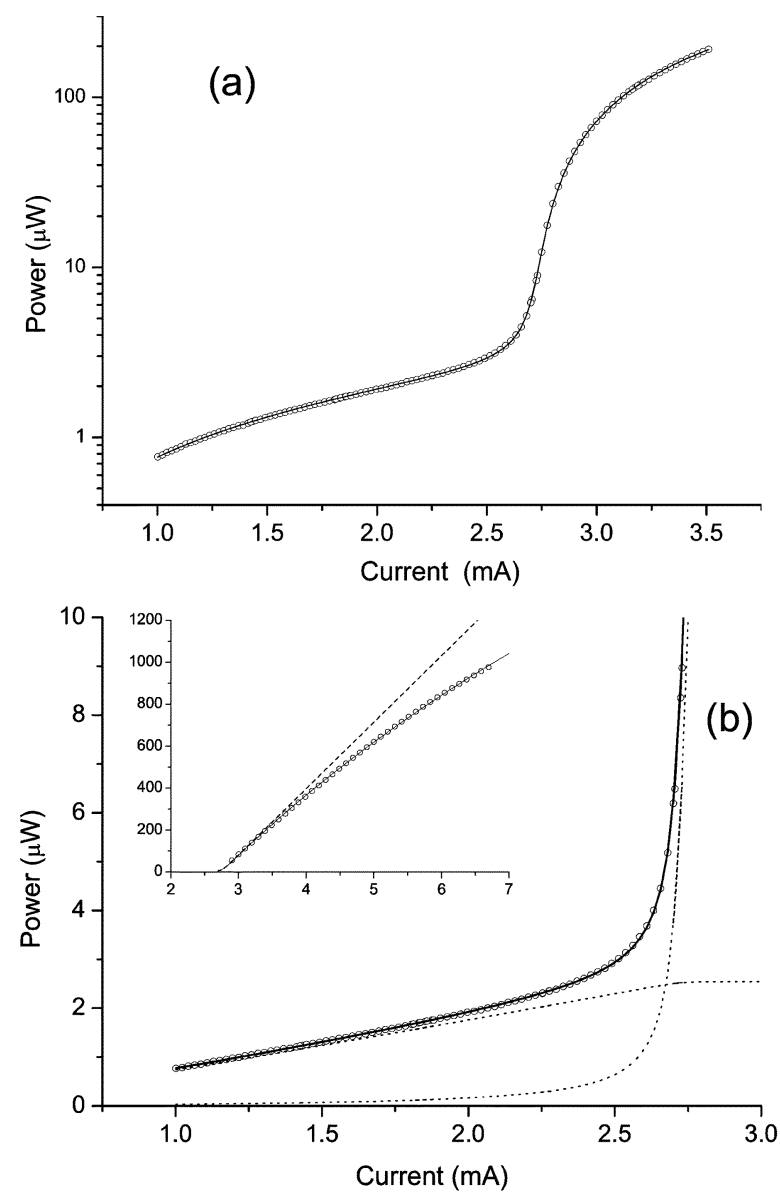

Fig. 1. Detected power as a function of the injection current. Open circles: experimental data. Solid line: fitting theoretical curve (see text). (a) Curves in the logarithmic scale. (b)Two different contributions in expression (37) are shown with dotted lines. The inset reports a wider current range, with the evidence of the thermal roll-off (in solid line the fitting curve, in dashed line the linear extrapolation).

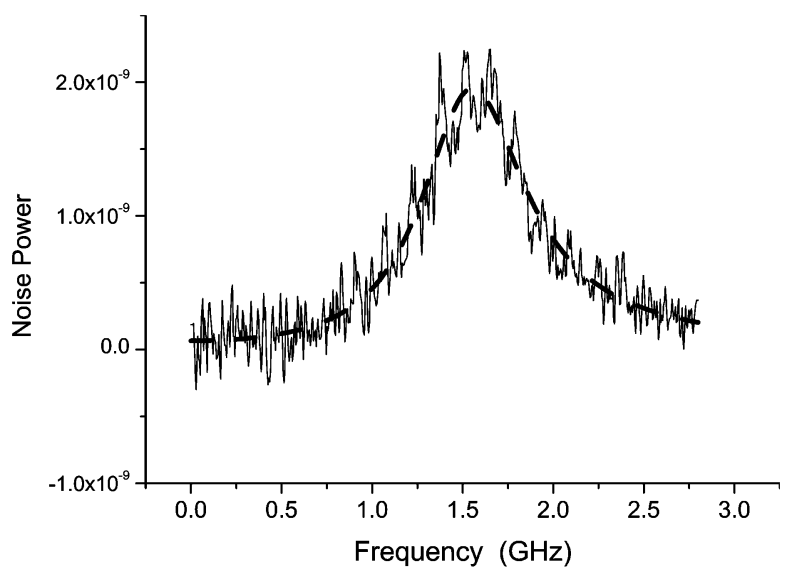

Fig. 2. Amplitude noise power spectrum for an injection current of $2.98 \mathrm{~mA}$. With a dashed line, the fit with expression (29) where we have forced $\Gamma_{\mathrm{RO}}=$ $\Gamma_{\mathrm{RO}}^{\prime}$.

an amplitude noise spectrum recorded by means of the $\mathrm{p}-\mathrm{i}-\mathrm{n}$ diode is shown in Fig. 2. The APD spectral response is calibrated with the light of an halogen lamp, giving shot-noise limited intensity noise above few $\mathrm{kHz}$. This correction gives a very flat equivalent response and reduces the possibilities of errors
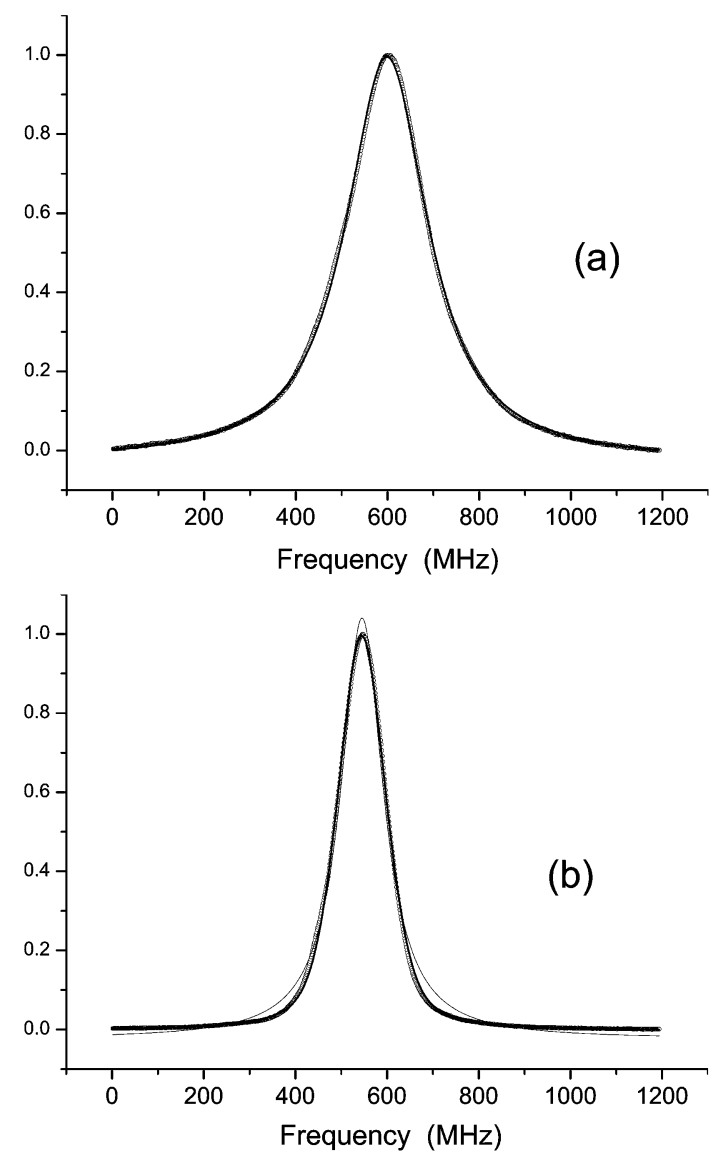

Fig. 3. Line profiles form the transmission of a Fabry-Pérot cavity. Open circles: experimental data. Solid lines: fitting functions. (a) For an injection current of $3.32 \mathrm{~mA}$, the fit is with a Lorentzian lineshape. (b) At $5.03 \mathrm{~mA}$, both a Lorentzian and a Voigt fitting profiles are shown.

due to distortion of the spectral shapes. Such a correction is not possible for the p-i-n photodiode due to its lower sensitivity. We have checked that the parameters obtained from the acquisitions of the two detector are in good agreement, in the region where the APD can be used (low currents).

The laser lineshape is measured from the transmission of a confocal Fabry-Pérot interferometer, which has a Free-Spectral Range of $2.5 \mathrm{GHz}$ and an instrumental linewidth of less than $10 \mathrm{MHz}$. Two examples, recorded at 3.32 and $5.03 \mathrm{~mA}$, are shown in Fig. 3.

\section{RESULTS AND DISCUSSION}

For reasonable values of the parameters, the current interval where $\Gamma_{\mathrm{RO}}$ is significantly different from $\Gamma_{\mathrm{RO}}^{\prime}$ is very narrow. In our case, it corresponds to few micro-amperes and it is difficult to obtain useful information from this region. For higher currents, we have verified with numerical simulations that an extremely high signal-to-noise ratio would be necessary to get reliable values of both $\Gamma_{\mathrm{RO}}$ and $\Gamma_{\mathrm{RO}}^{\prime}$ from a fitting procedure. On the other hand, a fit forcing $\Gamma_{\mathrm{RO}}^{\prime}=\Gamma_{\mathrm{RO}}$ in (29) gives accurate values of $\Omega_{\mathrm{RO}}$ and $\Gamma_{\mathrm{RO}}$. We have verified that, for the current region that we have considered, the results of such fit are correct within $1 \%$ for both parameters. Moreover, in the same region, $\Gamma_{\mathrm{RO}}^{\prime}$ differs from $\Gamma_{\mathrm{RO}}$ by less than $5 \%$, i.e., by less than 

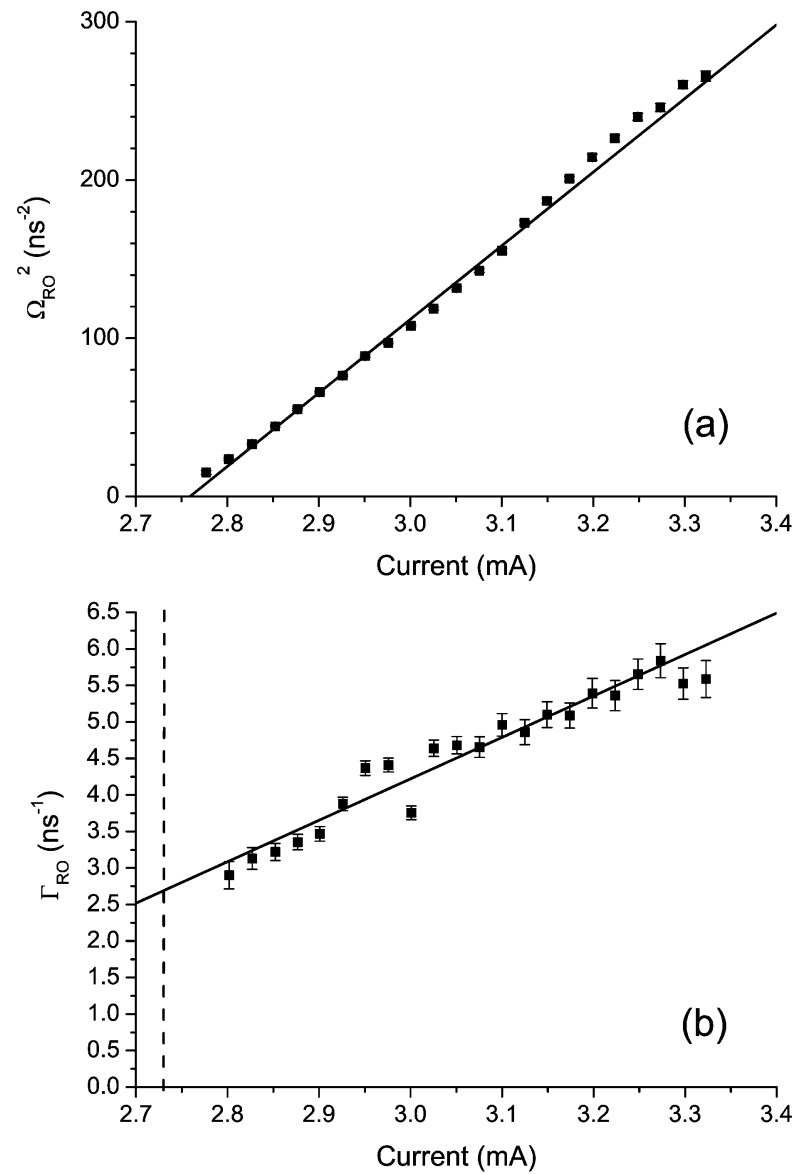

Fig. 4. Fitting parameters obtained from the study of the noise spectra, as a function of the injection current (symbols with statistic error bars given by each fit), together with linear interpolations (solid lines). The vertical dashed line shows the threshold current.

the experimental statistical uncertainty. Therefore, we will neglect in the following analysis the second term, proportional to $\widetilde{R}_{\mathrm{sp}}^{\mathrm{th}}$, in the expression (32) of $\Gamma_{\mathrm{RO}}$.

We have fitted the experimental RIN with a simplified expression (29) where $\Gamma_{\mathrm{RO}}^{\prime}=\Gamma_{\mathrm{RO}}$. An example is shown in Fig. 2 for $I=2.98 \mathrm{~mA}$. The fitting parameters $\Omega_{\mathrm{RO}}^{2}$ and $\Gamma_{\mathrm{RO}}$ are reported in Fig. 4 as a function of the pump current, together with their linear interpolations. The slope of $\Omega_{\mathrm{RO}}^{2}$ versus $\mu$ is $\left(\eta / \tau_{n} \tau_{p}\right)=1290 \pm 20 \mathrm{~ns}^{-2}$. The extrapolation to threshold of $\Gamma_{\mathrm{RO}}$ is $\left(1 / \tau_{n}\right)=2.69 \pm 0.14 \mathrm{~ns}^{-1}$, and the slope of $\Gamma_{\mathrm{RO}}$ versus $\mu$ is $\left(\eta / \tau_{n}\right)=15.5 \pm 1.3 \mathrm{~ns}^{-1}$. From these measurements we deduce $\tau_{n}=0.37 \pm 0.02 \mathrm{~ns}, \tau_{p}=12 \pm 1 \mathrm{ps}$, and $\eta=5.8$. We remark that the relaxation oscillations frequency is rather well defined, while the determination of the damping parameter, associated with the spectral width, is probably the most critical part of the whole procedure described in this paper.

The $L-I$ curve is reported in Fig. 1 in linear-logarithmic scale. Even limiting the observation to pump currents near and above threshold (e.g., above $2.4 \mathrm{~mA}$ ), the expression (33) does not fit well the data. This can be explained by considering that (33) is only valid for the light power in the lasing mode, while the detector also sees a fraction of the wideband spontaneous emission. It can be taken into account by fitting the data with the expression

$$
P_{\text {det }}=C_{1} \frac{\bar{P}}{1+s \bar{P}}+C_{2} \bar{n}^{2}
$$

where $P_{\text {det }}$ is the detected power and $C_{1}$ and $C_{2}$ are free constants. The denominator, with $s$ as free parameter, gives a small phenomenological correction accounting for the thermal rolloff, as detailed later.

A first approximated analytical expression for $P_{\text {det }}$ can be obtained well below threshold, where the stimulated emission can be neglected, the contribution of $\bar{P}$ to $P_{\text {det }}$ can be omitted and the (18) can be written in the form

$$
I=c_{1} \sqrt{P_{\mathrm{det}}}+c_{2} P_{\mathrm{det}}+c_{3} P_{\mathrm{det}}^{(3 / 2)} .
$$

Simple calculations comparing (38) with (18) show that the following relations hold:

$$
\begin{aligned}
\frac{c_{1}^{2}}{c_{2}} & =\frac{I_{\mathrm{th}} \widetilde{A}^{2}}{\widetilde{A}+1+\widetilde{C}} \\
\frac{c_{3}^{2}}{c_{2}^{3}} & =\frac{\widetilde{C}^{2}}{I_{\mathrm{th}}(\widetilde{A}+1+\widetilde{C})} .
\end{aligned}
$$

From the experimental parameters $c_{1}, c_{2}$, and $c_{3}$, we can get $\widetilde{A}$ and $\widetilde{C}$. We have used the (38) to fit the data below $1.9 \mathrm{~mA}$, finding that $\widetilde{C} \simeq 0$, i.e., that the Auger effect is negligible. This was indeed expected for a AlGaAs laser [23].

A further approximated expression for $P_{\text {det }}$ is obtained by using (33) for $\bar{P}$ and (28) for $\bar{n}$. Such expression could be used near and above threshold. It can be further extended to lower currents by using a more general solution for $\bar{n}$, that is derived from the complete (17) where we replace the solution (26) for $\bar{P}$. Indeed we can safely assume that (26) is valid in the current region where the stimulated emission plays an important role in the equation for $n$. If we also take $\widetilde{C}=0$ we can derive an analytical expression for $\bar{n}$ in (41), shown at the bottom of the page, that we have used in the (37) to fit the complete set of data. The resulting curve, shown in Fig. 1, agrees very well with the experiment (better than $3 \%$ on the whole range).

In Fig. 1(b) we also distinguish the contributions of $\bar{P}$ and $\bar{n}^{2}$ to $P_{\text {det. }}$. In the region around threshold, $\bar{n}^{2}$ is very flat and the shape of the knee is mostly determined by the factor $\left(\tau_{p} \widetilde{R}_{\mathrm{sp}}^{\mathrm{th}} / \eta^{2}\right)$ that we have put into evidence as one of the free parameters in our fitting procedure. Its value is, therefore, accurately obtained, and we found $(8.1 \pm 0.1) \cdot 10^{-5}$. In this derivation, the interesting region of the $L-I$ curve is the knee

$$
\bar{n}=\frac{n_{\mathrm{th}}}{2}\left[-\widetilde{A}-(2+\widetilde{A}) \bar{P}+\sqrt{[\widetilde{A}+(2+\widetilde{A}) \bar{P}]^{2}+4\left[(1+\widetilde{A}) \mu+(2+\widetilde{A}) \frac{1}{n_{\mathrm{th}}} \bar{P}\right]}\right]
$$


around threshold. Therefore, the temperature dependence of the threshold, whose importance in VCSELs is underlined in [22], is not critical due to the limited significant current interval.

The fit is very weakly dependent on $n_{\mathrm{th}}$, therefore, we have taken $\eta$ from the previous measurements on the RIN and fixed $n_{\text {th }}$, using the (13) with $\widetilde{C}=0$, as

$$
n_{\mathrm{th}}=\frac{1}{1-\frac{1}{\eta} \frac{1+\widetilde{A}}{2+\widetilde{A}}} .
$$

Finally, we remark that the obtained value of $\widetilde{A}=0.44$ (and, as a consequence, of $n_{\mathrm{th}}$ ) is mostly given by the slope of the $\bar{n}^{2}$ contribution below threshold.

Olshansky et al. [23] have shown in a AlGaAs LED that the coefficient $B$ is not exactly constant, giving a contribution to the radiative recombination which is cubic in the population density. Such term would contribute by about $10 \%$ to our spontaneous emission at threshold. Our experiment is not accurate enough to distinguish it, but we have verified numerically that the other parameters are not significantly affected by this uncertainty.

A deviation from linearity is clear at high current, as shown in the inset of Fig. 1(b), due to the detuning between lasing mode and gain peak (thermal roll-off). We account for it with a phenomenological saturation term, as shown in (37). This effect is only few per cent at $3.5 \mathrm{~mA}$.

From the fitted parameters and the measurements on the RIN we can easily calculate $\widetilde{R}_{\mathrm{sp}}^{\mathrm{th}}=(2.3 \pm 0.6) \cdot 10^{-4} \mathrm{ps}^{-1}$, i.e., $\tau_{p} \widetilde{R}_{\mathrm{sp}}^{\mathrm{th}}=(2.7 \pm 0.6) \cdot 10^{-3}$, and $n_{\mathrm{th}}=1.11 \pm 0.01$. The value of $n_{\text {th }}$ confirms that the carrier density at threshold is not far from transparency, and the linear expansion of the gain versus carrier density around transparency is well justified.

The analysis of the phase noise reported in the previous section only considers Schawlow-Townes phase diffusion due to spontaneous emission which gives a Lorentzian lineshape. However, it is shown in [8] that such a function can be inaccurate in reproducing the laser spectral shape, which is better described by a Voigt profile, i.e., by a convolution of a Lorentzian and a Gaussian function. The Gaussian contribution is due to excess low-frequency noise, that is indeed directly shown in [24]. We have found the same situation for our laser: a Lorentzian shape can well reproduce the data for low current values, as shown in Fig. 3(a), but at higher current the narrower line is not well described by the Lorentzian. We have fitted to the data the Whiting [25] approximation of the Voigt profile

$$
\begin{aligned}
f(\nu)=V_{0}\left\{\left(1-\frac{\gamma_{L}}{\gamma_{V}}\right) \exp [-\right. & \left.2.772\left(\frac{\nu-\nu_{0}}{\gamma_{V}}\right)^{2}\right] \\
+ & \left.\frac{\gamma_{L}}{\gamma_{V}} \frac{1}{1+4\left(\frac{\nu-\nu_{0}}{\gamma_{V}}\right)^{2}}\right\}
\end{aligned}
$$

where $V_{0}$ is the Voigt profile amplitude, $\nu_{0}$ is the central frequency, and $\gamma_{L}$ and $\gamma_{V}$ are the full linewidth (FWHM), respectively, of the Lorentzian and of the Voigt profile. The Whiting approximation is accurate within $1 \%$ in determining the Lorentzian linewidth, for our range of parameters. As shown in Fig. 3(b), this function fits much better the data. The parameter that must be compared with (35) is $\gamma_{L}$, which is

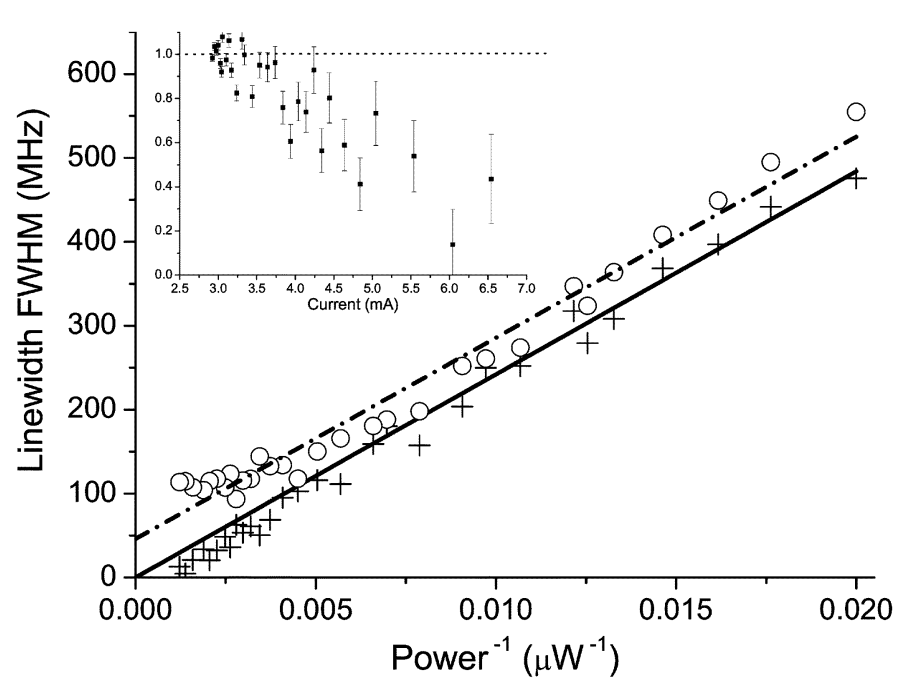

Fig. 5. Crosses: width of the Lorentzian component of the laser lineshape, as a function of the inverse of the power, together with a linear interpolation (solid line). Open symbols: full linewidth, interpolated by a dashed-dotted line. In the inset, the linewidth-power product is plotted as a function of the current, normalized to its low-power value of $24.2 \mathrm{MHz} \cdot \mathrm{mW}$. The error bars account for the uncertainty in the thermal roll-off correction to the power and in the instrumental width.

reported in Fig. 5 as a function of the inverse of the laser power. The results are very well fitted by a straight line, without the residual power-independent linewidth which is often observed even in VCSELs [4], [5], [26] and is difficult to model. We remark that such a result is due to the removal of the Gaussian component: the complete laser linewidth, also shown in Fig. 5, would have given a wrong slope and a misleading residual width. On the contrary, the $\gamma_{L}$ values fall slightly below the fitting line for high power. Therefore, we have inferred the slope by considering only the linewidth values above $130 \mathrm{MHz}$ and by taking a conservative uncertainty. The linewidth-power product is $24.2 \pm 0.4 \mathrm{MHz} \cdot \mathrm{mW}$, corresponding to $35.0 \mathrm{MHz}$ $\cdot 1 /(\mu-1)$ after the correction for the thermal roll-off.

The weak deviation at high power could be interpreted as a decrease of $\alpha$ (and/or of the spontaneous emission factor) at increasing current, maybe motivated by the change in the detuning between lasing frequency and gain peak. This is shown more clearly in the inset of Fig. 5, where the linewidth-power product is plotted as a function of the current, normalized to its low-power value of $24.2 \mathrm{MHz} \cdot \mathrm{mW}$. The data are rather spread at high current,, however, a systematic trend is clear. We remark that here the accuracy is poor, due to the relatively large Gaussian component and also to the uncertainty in the estimation of the thermal roll-off and of the Fabry-Pérot instrumental width that is subtracted to the measured $\gamma_{L}$. Therefore, our results must be considered as a possible indication of a current-dependent $\alpha$, not really a demonstration.

It is now useful to describe explicitly how the linewidth enhancement parameter $\alpha$ can be deduced from the above described measurements, to give a clear idea of how its accuracy depends from the single experimental results. We have

$$
1+\alpha^{2}=4 \pi\left[\Delta \nu_{S T}(\mu-1)\right] \times\left[\frac{\eta^{2}}{\tau_{p} \widetilde{R}_{\mathrm{sp}}^{\mathrm{th}}}\right] \times\left[\frac{\tau_{p} \tau_{n}}{\eta}\right] \times\left[\frac{1}{\tau_{n}}\right]
$$


TABLE I

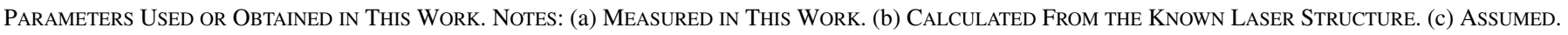
(d) DEDUCED From the Other PARAMETERS

\begin{tabular}{|c|c|c|c|c|}
\hline Parameter & Description & Value & Units & Notes \\
\hline$\tau_{n}$ & small signal carrier lifetime & $0.37 \pm 0.02$ & ns & $\mathrm{a}$ \\
\hline$\tau_{p}$ & photon lifetime & $12 \pm 1$ & ps & $\mathrm{a}$ \\
\hline$\eta$ & modified gain & $5.8 \pm 0.6$ & & $\mathrm{a}$ \\
\hline$\widetilde{R}_{s p}^{t h}$ & modal spontaneous emission rate & $(2.3 \pm 0.6) \cdot 10^{-4}$ & $\mathrm{ps}^{-1}$ & $\mathrm{a}$ \\
\hline$\widetilde{A}$ & see text (after Eq. 11) & $0.44 \pm 0.01$ & & $\mathrm{a}$ \\
\hline$\widetilde{B}$ & see text (after Eq. 11) & $1.23 \pm 0.07$ & $\mathrm{~ns}^{-1}$ & $\mathrm{a}$ \\
\hline$\widetilde{C}$ & see text (after Eq. 11) & $<0.2$ & & $\mathrm{a}$ \\
\hline$n_{t h}$ & normalized carrier density at threshold & $1.11 \pm 0.01$ & & $\mathrm{a}$ \\
\hline$\alpha$ & linewidth enhancement factor & $3.2 \pm 0.1$ & & $\mathrm{a}$ \\
\hline$w$ & modal waist & $2.0 \pm 0.1$ & $\mu \mathrm{m}$ & $\mathrm{a}$ \\
\hline$v_{G}$ & group velocity & $0.88 \cdot 10^{8}$ & $\mathrm{~m} / \mathrm{s}$ & b \\
\hline$r_{0}$ & mesa radius & 4.7 & $\mu \mathrm{m}$ & $\mathrm{b}$ \\
\hline$\Gamma_{l g}$ & longitudinal confinement factor & 0.041 & & $\mathrm{~b}$ \\
\hline$L_{a}$ & active region thickness & 24 & $\mathrm{~nm}$ & $\mathrm{~b}$ \\
\hline$N_{0}$ & carrier density at transparency & $1.24 \cdot 10^{18}$ & $\mathrm{~cm}^{-3}$ & $\mathrm{c}$ \\
\hline$V$ & effective mode volume & 0.3 & $\mu \mathrm{m}^{3}$ & $\mathrm{~d}$ \\
\hline$a$ & gain coefficient & $1.4 \cdot 10^{-15}$ & $\mathrm{~cm}^{2}$ & $\mathrm{~d}$ \\
\hline$\beta$ & spontaneous emission factor & $1.1 \cdot 10^{-4}$ & & $\mathrm{~d}$ \\
\hline$R_{s p}$ & spontaneous emission rate (at threshold) & 0.3 & $\mathrm{ps}^{-1}$ & $\mathrm{~d}$ \\
\hline$A$ & see text (after Eq. 5) & 0.49 & $\mathrm{~ns}^{-1}$ & $\mathrm{~d}$ \\
\hline$B$ & see text (after Eq. 5) & $7.8 \cdot 10^{-19}$ & $\mathrm{~cm}^{3} \mathrm{~ns}^{-1}$ & $\mathrm{~d}$ \\
\hline$C$ & see text (after Eq. 5) & $<10^{-37}$ & $\mathrm{~cm}^{6} \mathrm{~ns}^{-1}$ & $\mathrm{~d}$ \\
\hline
\end{tabular}

where in the above product we have factorized the directly measured parameters. The result is $\alpha=3.2 \pm 0.1$, where the uncertainties have been summed quadratically.

From the measured functional parameters we can now derive some further information on the laser physics. At this purpose, we have calculated some parameters from the known laser structure (namely, $v_{G}, r_{0}, \Gamma_{l g}, L_{a}$, reported in Table I). Moreover, we assume a carrier density at transparency of $N_{0}=$ $1.24 \cdot 10^{18} \mathrm{~cm}^{-3}$. We have also evaluated from the laser beam propagation a waist of $w=2.0 \pm 0.1 \mu \mathrm{m}$, from which we calculate a transverse confinement factor of $\Gamma_{t r}=0.97$ and an effective volume $V=0.3 \mu \mathrm{m}^{3}$.

From (8) we deduce a gain coefficient $a=1.4 \cdot 10^{-15} \mathrm{~cm}^{2}$. The (12) gives $\widetilde{B}=1.23 \mathrm{~ns}^{-1}$ and from the definition of $\widetilde{A}, \widetilde{B}$, $\widetilde{C}$ we deduce $A, B, C$. From (25) we obtain the spontaneous emission factor $\beta=1.1 \cdot 10^{-4}$.

A summary of the parameters measured or deduced in this work are reported in Table I.

As stated in Section II, we have neglected in our theoretical analysis the gain saturation, that is often relevant in semiconductor lasers. Its most important effect would be an additional term on $\Gamma_{\mathrm{RO}}$ (but not on $\Gamma_{\mathrm{RO}}^{\prime}$ ), proportional to $\mu / \tau_{p}$. Such term could be disclosed by the analysis of the RIN, from a difference between $\Gamma_{\mathrm{RO}}$ and $\Gamma_{\mathrm{RO}}^{\prime}$. We saw no such difference in our data, but, as already discussed, determining $\Gamma_{\mathrm{RO}}^{\prime}$ from the RIN is very difficult and additional measurements would be necessary for an accurate evaluation of the saturation effect. If present, it would imply smaller $\eta$ and $\tau_{p}$, without affecting $\alpha$.

\section{CONCLUSION}

We have presented a complete experimental evaluation of the effective parameters necessary to describe the dynamical behavior of an air-post VCSEL, on the basis of theoretical equations which are also derived in this paper. The experimental investigation is composed of several steps, including power versus current measurement, noise spectrum analysis, linewidth evaluation. Even if examples of the different studies are rather common in the literature (see, e.g., for early measurements in VCSELs, [4], [22]), it is in our knowledge the first time that the complete procedure is defined and tested, ${ }^{2}$ allowing the complete experimental determination of all the parameters.

\footnotetext{
${ }^{2}$ Similar experimental investigation is reported in [27].
} 
We have used these results to simulate numerically an optical feedback experiment using the same laser sample, and the exact determination of the parameters (in particular, the linewidth enhancement factor $\alpha$ and the spontaneous emission $\widetilde{R}_{\mathrm{sp}}^{\mathrm{th}}$ ) has proved to be extremely useful in the attempt of quantitatively reproduce the experimental findings. These results will be reported in a further publication.

\section{ACKNOWLEDGMENT}

The authors would like to thank G. Van der Sande for careful reading of the manuscript. Useful discussions within the framework of the European COST action 288 are acknowledged.

\section{REFERENCES}

[1] C. H. Henry, "Theory of the linewidth of semiconductor lasers," IEEE J. Quantum Electron., vol. 18, no. 2, pp. 259-264, Feb. 1982.

[2] M. Osiński and J. Buus, "Linewidth broadening factor in semiconductor lasers-An overview," IEEE J. Quantum Electron., vol. 23, no. 1, pp. 9-29, Jan. 1987.

[3] Y. Yu, G. Giuliani, and S. Donati, "Measurements of the linewidth enhancement factor of semiconductor lasers based on the optical feedback self-mixing effect," IEEE Photon. Technol. Lett., vol. 16, no. 4, pp. 990-992, Apr. 2004.

[4] B. Möller, E. Zeeb, U. Fiedler, T. Hackbarth, and K. J. Ebeling, "Linewidth enhancement factor of vertical-cavity surface-emitting laser diodes," IEEE Photon. Technol. Lett., vol. 6, no. 8, pp. 921-923, Aug. 1994.

[5] N. M. Margalit, J. Piprek, S. Zhang, D. I. Babic, K. Streubel, R. P. Mirin, J. R. Wesselmann, J. E. Bowers, and E. L. Hu, " $64{ }^{\circ} \mathrm{C}$ continuous-wave operation of $1.5-\mu \mathrm{m}$ vertical-cavity laser," IEEE J. Sel. Topics Quantum Electron, vol. 3, no. 2, pp. 359-365, Apr. 1997.

[6] P. Signoret, F. Marin, S. Viciani, G. Belleville, M. Myara, J. P. Tourrenc, B. Orsal, A. Plais, F. Gaborit, and J. Jacquet, "3.6 MHz linewidth 1.55 $\mu \mathrm{m}$ monomode vertical-cavity surface-emitting laser," IEEE Photon. Technol. Lett., vol. 13, no. 4, pp. 269-271, Apr. 2001.

[7] M. B. Willemsen, A. S. van de Nes, M. P. van Exter, J. P. Woerdman, M. Kicherer, R. King, R. Jäger, and K. J. Ebeling, "Polarization-resolved linewidth-power product of a vertical-cavity semiconductor laser," $J$. Appl. Phys., vol. 89, pp. 4183-4185, 2001.

[8] S. Viciani, M. Gabrysch, F. Marin, F. Monti di Sopra, M. Moser, and K. H. Gulden, "Lineshape of a vertical cavity surface emitting laser," Opt. Commun., vol. 206, pp. 89-97, 2002.

[9] A. L. Schawlow and C. H. Townes, "Infrared and optical masers," Phys. Rev., vol. 112, pp. 1940-1949, 1958.

[10] C. Risch and C. Voumard, "Self-pulsation in the output intensity and spectrum of GaAs-AlGaAs CW diode lasers coupled to a frequencyselective external optical cavity," J. Appl. Phys., vol. 48, pp. 2083-2085, 1977.

[11] G. Giacomelli, F. Marin, and M. Romanelli, "Multi-time-scale dynamics of a laser with polarized optical feedback," Phys. Rev. A, vol. 67, pp. 053 809-053 809, 2003.

[12] G. Vaschenko, M. Giudici, J. J. Rocca, C. S. Menoni, J. R. Tredicce, and S. Balle, "Temporal dynamics of semiconductor lasers with optical feedback," Phys. Rev. Lett., vol. 81, pp. 5536-5539, 1998.

[13] M. C. Soriano, M. Yousefi, J. Danckaert, S. Barland, M. Romanelli, G. Giacomelli, and F. Marin, "Low-frequency fluctuations in vertical-cavity surface-emitting lasers with polarization selective feedback: Experiment and theory," IEEE J. Sel. Topics Quantum Electron., vol. 10, no. 5, pp. 998-1005, Sep./Oct. 2004.

[14] A. V. Naumenko, N. A. Loiko, M. Sorendmann, and T. Ackemann, "Description and analysis of low-frequency fluctuations in vertical-cavity surface-emitting lasers with isotropic optical feedback by a distant reflector," Phys. Rev. A, vol. 68, pp. 033 805-033 805, 2003.

[15] A. Valle and L. Pesquera, "Analytical calculation of transverse mode characteristics in vertical-cavity surface-emitting lasers," J. Opt. Soc. Amer., vol. 19, pp. 1549-1557, 2002.

[16] P. Debernardi, L. Fratta, and G. P. Bava, "Spectral linewidth in oxideconfined vertical-cavity surface-emitting lasers," IEEE J. Quantum Electron., vol. 37, no. 8, pp. 1084-1094, Aug. 2001.

[17] K. Petermann, Laser Diode Modulation and Noise. Norwell, MA: Kluwer, 1991.
[18] A. Valle and L. Pesquera, "Theoretical calculation of relative intensity noise of multimode vertical-cavity surface-emitting lasers," IEEE J. Quantum Electron., vol. 40, no. 6, pp. 597-606, Jun. 2004.

[19] K. H. Gulden, M. Moser, S. Lüscher, and H. P. Schweizer, "High performance near-IR (765 nm) AlAs/AlGaAs vertical cavity surface emitting lasers," Electron. Lett., vol. 31, pp. 2176-2177, 1995.

[20] F. Monti di Sopra, "Optical properties of VCSELs and phase-coupled VCSEL arrays," Ph.D. dissertation, EPFL, Lausanne, Switzerland, 2001.

[21] M. Moser, Avalon Photonics Ltd., Zurich, Switzerland, private communication, May 10, 2004.

[22] Y. G. Zhao, J. G. McInerney, and R. A. Morgan, "Measurement of spontaneous emission factor for vertical-cavity surface-emitting semiconductor lasers," IEEE Photon. Technol. Lett., vol. 7, no. 11, pp. 1231-1233, Nov. 1995.

[23] R. Olshansky, C. B. Su, J. Manning, and W. Powazinik, "Measurement of radiative and nonradiative recombination rate in InGaAsP and $\mathrm{AlGaAs}$ light sources," IEEE J. Quantum Electron., vol. 20, no. 8, pp. 838-854, Aug. 1984.

[24] F. Marin and S. Viciani, "Frequency noise and lineshape of VCSELs," presented at the SPIE Photonics West Conf., vol. 4286, 2001, paper No. 4286-15.

[25] E. E. Whiting, "An empirical approximation to the voigt profile," $J$. Quant. Spectrosc. Radiat. Transf., vol. 8, pp. 1379-1384, 1968.

[26] F. Monti di Sopra, H. P. Zappe, M. Moser, R. Hövel, H. P. Gauggel, and K. Gulden, "Near-infrared vertical-cavity surface-emitting lasers with 3-MHz linewidth," IEEE Photon. Technol. Lett., vol. 11, no. 12, pp. 1533-1535, Dec. 1999.

[27] F. Robert, "Controle de L'état de polarization d'un laseràémission par la surface par l'application d'une contre-réaction optique," Ph.D. dissertation, Univ. Rennes I, Rennes, France, 1997.

Stephane Barland obtained the M.S. and Ph.D. degrees in physics from the Université de Nice, Nice, Italy, in 1998 and 2001, respectively.

His specialization field is nonlinear dynamics in optical systems. He worked at IMEDEA, Palma de Mallorca, Spain, on all-optical information processing and with the Istituto Nazionale per la Fisica della Materia (INFM), Unità di Firenze, 50019 Fiorentino, Italy, on semiconductor lasers. He is now full-time Researcher with the Institut Non-Linéaire de Nice, Valbonne, France .

Piernicola Spinicelli received the M.S. degree in physics from the University of Florence, Florence, Italy, in 2004 with a work on the characterization of verticalcavity semiconductor lasers.

He is currenty with the Physics Department, University of Florence, Florence, Italy.

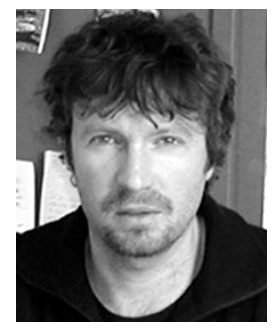

Giovanni Giacomelli was born in Florence, Italy, in 1962. He received the M.S. degree in physics from the University of Florence, in 1989 and the Ph.D. degree at the School of Optics, Florence, in 1992.

In 1994, he was a Researcher with the Istituto Nazionale di Ottica Applicata (INOA), Florence. He is now with the Instituto Sistemi Complessi-CNR, where he is the principle responsible of the SPICE project. His main research interests are the experimental study of instabilities in dynamical systems and the experimental and theoretical study of delayed

dynamical systems.

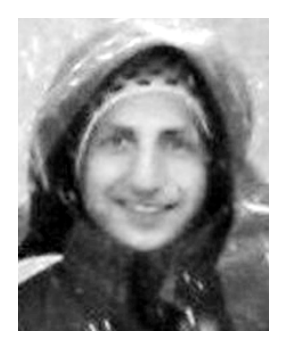

Francesco Marin was born in 1966. He received the M.S. degreefrom the University of Pisa, Pisa, Italy, in 1990, and the Ph.D. degree from the Scuola Normale Superiore of Pisa, Pisa, in 1994, both in physics.

$\mathrm{He}$ was a full-time Researcher at the Physics Department of the University of Florence, Florence, Italy, from 1998 to 2002, where he has been a Professor of physics since 2002. He published about 50 articles in the fields of atomic and molecular spectroscopy, quantum optics, spicy cooking, and laser physics. 